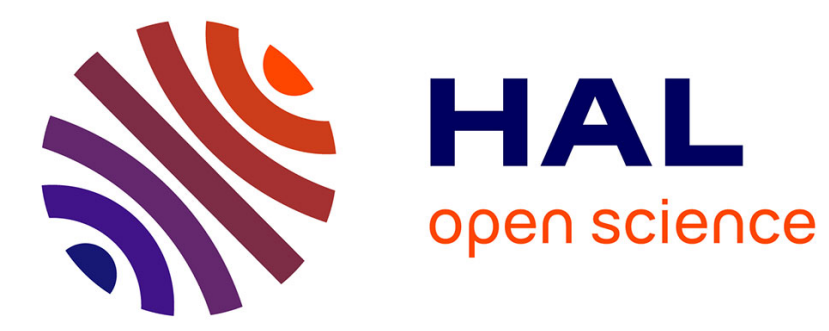

\title{
Numerical identification of linear cracks in 2D elastodynamics using the instantaneous reciprocity gap
}

\author{
Huy Duong Bui, Andrei Constantinescu, Hubert Maigre
}

\section{To cite this version:}

Huy Duong Bui, Andrei Constantinescu, Hubert Maigre. Numerical identification of linear cracks in 2D elastodynamics using the instantaneous reciprocity gap. Inverse Problems, 2004, 20, pp.993-1001. 10.1088/0266-5611/20/4/001 . hal-00111414

\author{
HAL Id: hal-00111414 \\ https://hal.science/hal-00111414
}

Submitted on 28 Feb 2019

HAL is a multi-disciplinary open access archive for the deposit and dissemination of scientific research documents, whether they are published or not. The documents may come from teaching and research institutions in France or abroad, or from public or private research centers.
L'archive ouverte pluridisciplinaire $\mathbf{H A L}$, est destinée au dépôt et à la diffusion de documents scientifiques de niveau recherche, publiés ou non, émanant des établissements d'enseignement et de recherche français ou étrangers, des laboratoires publics ou privés. 


\title{
Numerical identification of linear cracks in 2D elastodynamics using the instantaneous reciprocity gap
}

\author{
Huy Duong Bui, Andrei Constantinescu and Hubert Maigre \\ Laboratoire de Mécanique des Solides (CNRS UMR 7649), Ecole Polytechnique, \\ 91128 Palaiseau Cedex, France \\ E-mail: bui@lms.polytechnique.fr, constant@lms.polytechnique.fr and \\ maigre@1ms.polytechnique.fr
}

\begin{abstract}
This paper considers the identification problem of a linear crack in a body of finite extension within the framework of linear two-dimensional elastodynamics. In a series of prior papers in electricity, elasticity or acoustics, it has been proved using the reciprocity gap that three different series of adjoint wave fields determine in closed-form solution the normal of the plane of the crack, the position of the plane and finally the complete crack extension. The work developed next within the framework of linear elastodynamics defines a novel instantaneous reciprocity gap as the instantaneous work done by the adjoint tractions on the crack opening displacement. This quantity is then used to identify linear cracks in a two-dimensional problem. It is shown using a numerical example that a unique family of planar shear waves permits the identification of the normal, position and a convex hull of a linear crack through simple interpretations of the instantaneous reciprocity gap. This method is more general in the sense that it applies to three-dimensional problems as well.
\end{abstract}

(Some figures in this article are in colour only in the electronic version)

\section{Introduction}

The analysis of the diffraction of elastodynamic waves by a crack in a solid body in order to reconstruct the crack is a classical inverse problem. The problem is of interest to areas such as nondestructive testing or earthquake engineering.

The classical investigations of this inverse problem found in the literature (see, for example, [3]) are subject to different limitations: unbounded domain, domain with known Green function, source in the interior of the body, source far away from the crack in order to consider plane waves, etc. 
In recent years a series of theoretical results has been obtained on the dynamic problem or on similar time-dependent crack identification problems using the optimal control technique [7], or by constructing an appropriate adjoint field and applying the method of the reciprocity gap [5].

The papers presenting planar crack identification using the reciprocity gap [1, 2] constructed generally three families of adjoint waves which permitted us to identify in order the normal of the plane, its position and finally the complete extension of the crack.

The purpose of this paper is not the proposition of three new families of adjoint waves suited to identify the planar crack in the elastodynamic case. Its novelty consists of the definition of an instantaneous reciprocity gap, which is the instantaneous mechanical work done by the virtual tractions given by the adjoined field and the crack opening displacement. A simple interpretation of this result permits the determination of the normal and the position of the crack plane, as well as of a 'convex approximation' of the crack.

The paper starts with an introduction of the direct and the inverse problems in a general three-dimensional framework. The next section presents the reciprocity gap in the case of the general elastodynamic and the definition of the instantaneous reciprocity gap. Then a numerical example for the identification of a linear crack in a two-dimensional body is given. The identification results are obtained by a simple interpretation of the instantaneous reciprocity gap. The proposed technique is not dependent on the two-dimensional character of the problem and can therefore be easily extended to three-dimensional problems. The final section shows that the specific boundary condition occurring during slip faults in earthquakes is compatible with the present method and indicates as such a field of applications for this technique.

\section{The direct and the inverse problem}

Let us consider a homogeneous elastic body $\Omega$, containing a crack $\Gamma_{C}$ in its interior. We shall suppose that the crack $\Gamma_{C}$ is included in a plane $\Pi$. The exterior boundary of $\Omega$ will be denoted by $\partial \Omega$.

For each time instant $t \in[0, \infty)$, we shall consider that the vector field of displacements $\boldsymbol{u}$ and the tensor fields of strains and respectively stresses $\epsilon, \boldsymbol{\sigma}$ satisfy the following system of equations:

$$
\operatorname{div} \sigma=\rho \ddot{\boldsymbol{u}} \quad \boldsymbol{\sigma}=\boldsymbol{C} \varepsilon \quad \varepsilon=\frac{1}{2}\left(\nabla+\nabla^{T}\right) \boldsymbol{u}
$$

which is equivalent to the hyperbolic elastodynamic equation:

$$
\operatorname{div} \boldsymbol{C} \nabla \boldsymbol{u}=\rho \ddot{\boldsymbol{u}}
$$

where $C$ denotes the fourth-order tensor of elastic moduli. $C$ is supposed to be positive definite and to respect the classical symmetry relations:

$$
C_{i j k l}=C_{k l i j}=C_{i j l k} \text {. }
$$

We assume that both displacements and tractions are known on the exterior boundary $\partial \Omega$ for each time instant $t \in[0, \infty)$ :

$$
\left.\boldsymbol{u}\right|_{\partial \Omega}=\left.\boldsymbol{\xi} \quad \boldsymbol{\sigma} \cdot \boldsymbol{n}\right|_{\partial \Omega}=\phi
$$

where by $\boldsymbol{\sigma}=\boldsymbol{C} \nabla \boldsymbol{u}$ we have denoted the tensor field of stresses.

The two lips of the cracks $\Gamma_{C}^{+}$and $\Gamma_{C}^{-}$are supposed to be stress free:

$$
\left.\boldsymbol{\sigma} \cdot \boldsymbol{n}\right|_{\Gamma_{C}^{ \pm}}=0 \text {. }
$$

These boundary conditions are completed by a series of initial conditions on the displacement and the velocity fields. 
We remark that in the most practical case, we shall have a free surface $\phi=\mathbf{0}$, meaning that tractions have not to be measured and that displacements $\boldsymbol{\xi}$ are measured in a finite number of locations generally using accelerometers. This is, for example, the case of the free surface of the earth when modelling earthquakes. A more precise discussion of the boundary conditions and their importance with respect to earthquake problems is given in section 5 .

\section{The instantaneous reciprocity gap}

The variational principle associated with equation (2) permits after a series of integrations by parts and application of the boundary condition for the solution to define the adjoint problem:

$$
\operatorname{div} \boldsymbol{C} \nabla \boldsymbol{w}=\rho \ddot{\boldsymbol{v}}
$$

and the reciprocity gap:

$$
\begin{aligned}
\mathcal{R B}= & \int_{0}^{\infty} \int_{\Gamma_{C}} \llbracket \boldsymbol{u} \rrbracket \cdot \boldsymbol{\sigma}[\boldsymbol{w}] \cdot \boldsymbol{n} \mathrm{d} s \mathrm{~d} t \\
= & \int_{0}^{\infty} \int_{\partial \Omega}\{\boldsymbol{u} \cdot \boldsymbol{\sigma}[\boldsymbol{w}] \cdot \boldsymbol{n}-\boldsymbol{w} \cdot \boldsymbol{\sigma}[\boldsymbol{u}] \cdot \boldsymbol{n}\} \mathrm{d} s \mathrm{~d} t \\
& +\int_{\Omega \backslash \Gamma_{C}}\left[\boldsymbol{u} \cdot \partial_{t} \boldsymbol{w}-\partial_{t} \boldsymbol{u} \cdot \boldsymbol{w}\right]_{0}^{\infty} \mathrm{d} v .
\end{aligned}
$$

In the definition of the reciprocity gap, $\boldsymbol{u}$ is a solution of the direct problem (2) and directly related to the measurements, whether $\boldsymbol{w}$ is a solution of the adjoint problem (6) and arbitrarily fixed.

The instantaneous reciprocity gap will be simply defined by the following expression:

$$
\begin{aligned}
\mathcal{R B}(t) & =\int_{\Gamma_{C}} \llbracket \boldsymbol{u} \rrbracket \cdot \boldsymbol{\sigma}[\boldsymbol{w}] \cdot \boldsymbol{n} \mathrm{d} s \\
& =\int_{\partial \Omega}\{\boldsymbol{u} \cdot \boldsymbol{\sigma}[\boldsymbol{w}] \cdot \boldsymbol{n}-\boldsymbol{w} \cdot \boldsymbol{\sigma}[\boldsymbol{u}] \cdot \boldsymbol{n}\} \mathrm{d} s .
\end{aligned}
$$

Defined as such, the instantaneous reciprocity gap actually measures the instantaneous virtual mechanical work done on the crack tips by the real displacements on the adjoint traction field.

If the adjoint field is an adjoined wavefront, it follows that the instantaneous reciprocity gap is zero, unless the adjoint wavefront has reached the crack. As a consequence one can determine the arrival time of the adjoint wavefront at the crack from boundary measurements. The precise knowledge of the propagation velocity and the direction of the adjoint wavefront permits us then to determine the location of the crack. A series of techniques to practically exploit this observation will be clarified in the next section using a two-dimensional numerical example.

\section{Analysis of the numerical results}

The example considered here corresponds to the body presented in figure 1 . The numerical computations have been done in two steps.

In the first step we have been simulating a direct elastodynamic problem, and obtained a set of 'artificial' displacement measurements. The loading is an initial slip on the crack and is similar to those obtained in earthquakes. 


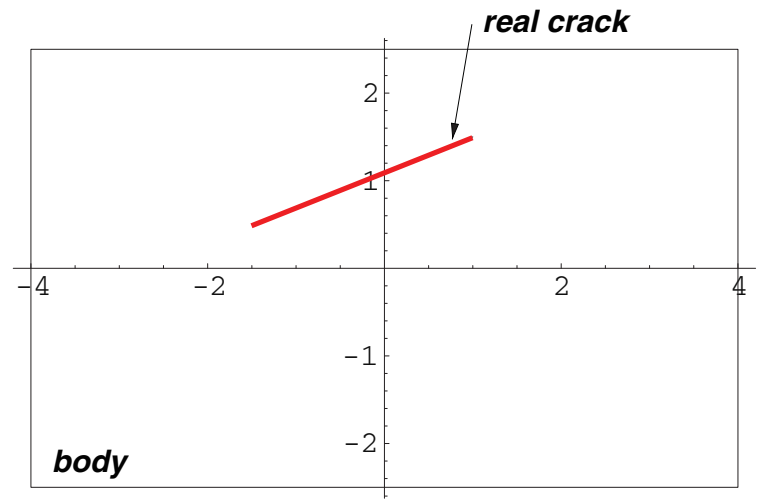

Figure 1. The geometry of the body and the position of the 'real' crack.

In the second step, these measurements have been convoluted with a series of adjoined fields and the results have been interpreted in order to identify the crack.

The direct computations and part of the identification procedure have been programmed using the Cast3M finite element programming language. The complete mesh had 800 nodes and 1500 linear triangular elements. The crack lips have been represented by 10 linear elements. A second-order algorithm has been used for the numerical integration direct wave propagation problem.

The loading of the direct problem has been given a tangential shear vector on the crack lips, which will be completely released after the initial moment $t=0$, i.e.:

$$
\tau(t)=\alpha Y(-t) t
$$

where $\alpha$ is constant, $Y$ is the unit step function and $t$ is the tangent vector on $\Gamma_{C}$.

All exterior surfaces are considered as stress free:

$$
\left.\boldsymbol{\sigma} \cdot \boldsymbol{n}\right|_{\partial \Omega}=0 \quad \text { i.e. } \phi=0 .
$$

The boundary displacement has been collected in the direct problem and considered in the identification part as a measurement.

These loading conditions slightly open the crack and lead to a large tangential slip when compared to the overlapping of the crack faces. From a practical point of view, it can be shown that this loading type is similar to that of an earthquake, owing to the large amount of slip, but this will not be detailed here. However, in the next section, we shall discuss in detail the effects of nonoverlapping contact boundary conditions on the instantaneous reciprocity gap.

The adjoint wave fields were constructed as 36 planar share wave fields. All directions of propagation of the wave fields were passing through the centre of the body and were equally spaced every $2 \pi / 36$ radians.

Moreover, the initial time for the adjoined waves was chosen such that all reach the centre of the body at the same instant.

$$
\boldsymbol{w}(\boldsymbol{x}, t)=\boldsymbol{k} Y\left(t-\frac{1}{c} \boldsymbol{x} \cdot \boldsymbol{p}-\tau\right) \boldsymbol{k}
$$

where $\boldsymbol{x}$ and $t$ denote the space point and time instant when the displacement $\boldsymbol{w}$ is computed. $p, k$ are the direction of propagation of the wave and respectively the direction of shear and are for each wave orthogonal: $\boldsymbol{p} \cdot \boldsymbol{k}=0 . \tau$ is a time parameter chosen such that the shear 


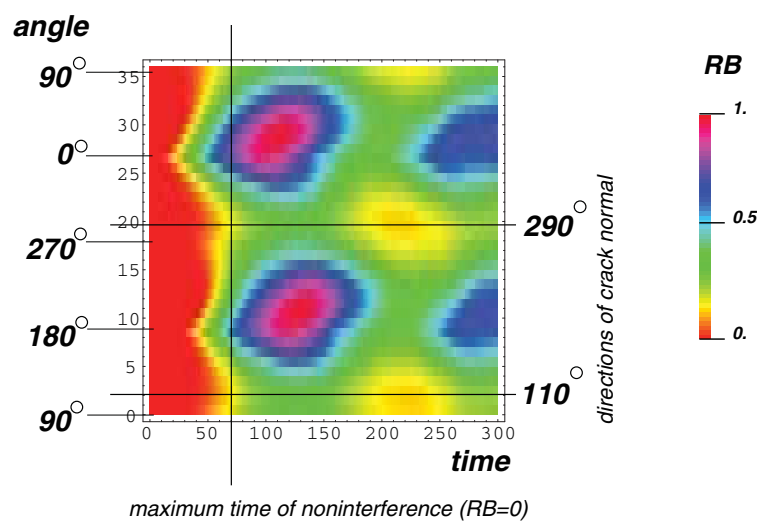

Figure 2. A plane colour plot representation of the time evolution ( $x$-axis) of the instantaneous reciprocity gap for different angles ( $y$-axis). The angles with the maximum noninterference of the adjoint wave with the crack define the direction of the normal (up to $180^{\circ}$ ).

wave is outside the body at the initial instant $t=O . c$ denotes the velocity of the propagation of the shear waves and equals

$$
c=\sqrt{\frac{E}{\rho(1+v)}}
$$

for an isotropic elastic body with Young modulus $E$, Poisson coefficient $v$ and a mass density $\rho$.

The stress state corresponding to the adjoined wave is a travelling Dirac impulse. It therefore does not produce any mechanical work with the displacement jump $\llbracket u \rrbracket$ on the crack unless the adjoined wave field has 'arrived' at the crack $\Gamma$ at time $t_{p}$. Therefore the instantaneous reciprocity gap vanishes for $t<t_{p}$ and has a nonzero value after this instant (see figure 3).

This is a consequence of the fact that the wavefront has to arrive at the crack in order to produce virtual work on it.

Next, using the previous remark on the interpretation of the instantaneous reciprocity gap and the fact that the adjoined waves are plane, we will be able to identify certain characteristics of the crack:

\section{- normal of the crack}

From simple geometric reasoning one can deduce that the adjoint wave having its wavefront parallel to the crack tips will interact with the crack at a later instant when compared to the wave coming from the same half-space with respect to the crack. Figure 2 reproduces the value of the instantaneous reciprocity gap at each time step ( $x$-axis) for each adjoined wave ordered by the incoming angle ( $y$-axis). In this plot one can remark that the angles of the adjoined wave for which the instantaneous reciprocity stays initially the longest period at the zero value, meaning no interference with the crack, are $110^{\circ}$ and $290^{\circ}$. They are $180^{\circ}$ apart, as they only indicate the direction of the normal to the crack.

- position of the crack plane

Assuming that the normal of the plane is known, let us analyse the instantaneous reciprocity gap obtained from the adjoined waves propagating in the direction of the normal and denote $t_{f}, t_{b}$ as the moments when the adjoint waves begin their interaction 


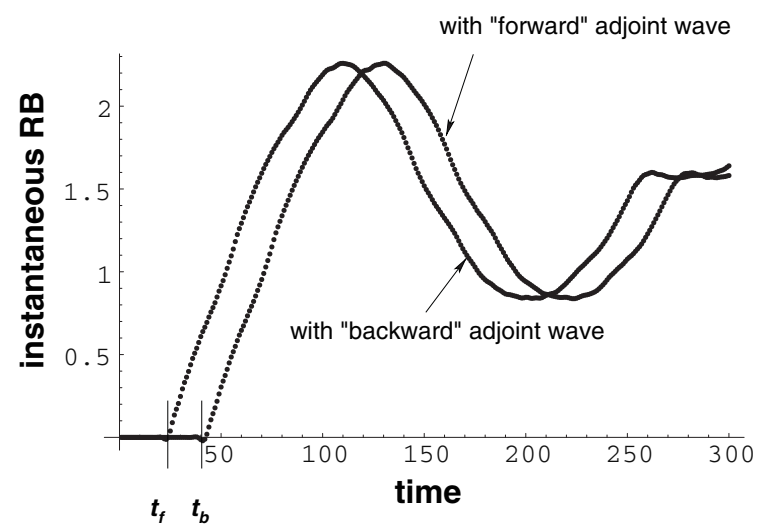

Figure 3. Time evolution of the computed reciprocity gap computed using two adjoined fields arriving at opposite directions called the backward (at $290^{\circ}$ ) and forward directions $\left(\right.$ at $\left.110^{\circ}\right)$. The arrival times of the adjoint wavefronts on the crack are denoted by $t_{b}$ and $t_{f}$ respectively.

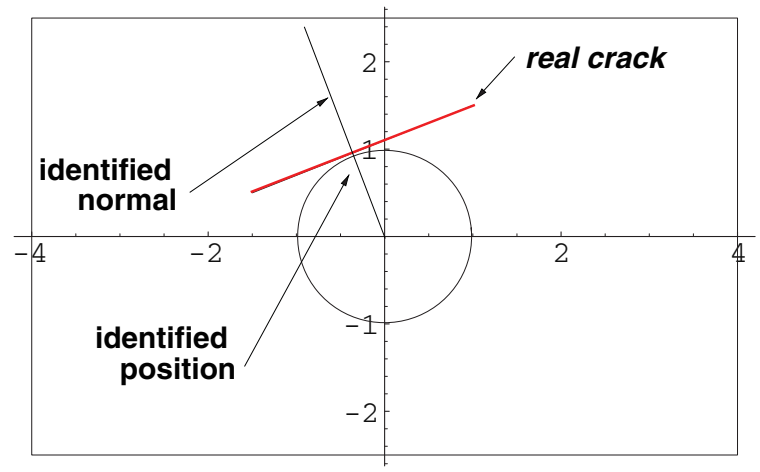

Figure 4. The real crack and its identified normal and position given by intersection of the normal with the circle.

with the crack. If $R$ represents the distance from the starting point of the adjoint waves to the centre of the body, we have

$$
c\left(t_{f}+t_{b}\right)=2 R
$$

where $v$ is the propagation speed of the wave. Then the distance of the crack to the centre of the body is given by either

$$
d=R-t_{f} c \quad \text { or } \quad d=R+t_{b} c .
$$

The results of this analysis are represented in figure 4 and compared with the initial position of the crack.

Another way to analyse the results of the instantaneous reciprocity gap is the following one. On the rays, characterizing the direction of propagation of the adjoint waves we represent at each time instant, in the real position of the wavefront, a segment representing the wavefront. The value of the instantaneous reciprocity gap is represented by a colour code (see figures 5 and 6). We recall that the evolution of the values of the instantaneous reciprocity gap on each line is similar to the graphs displayed in figure 3. The plot of the wave position is stopped when the reciprocity is larger than a small predefined value. The coloured domain represented 


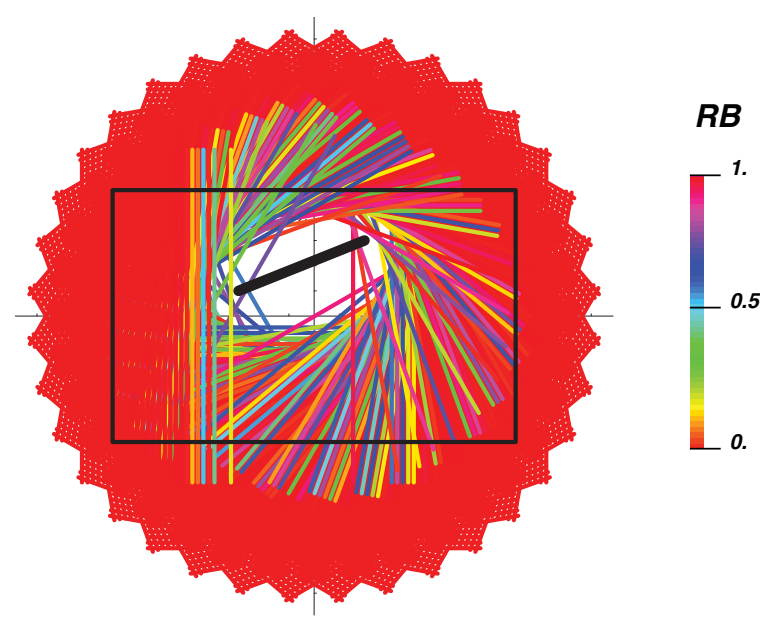

Figure 5. The position of the real crack and the 'convex hull' obtained using the front of the adjoint wave and the instantaneous reciprocity gap. Each line segment represents the position of the wavefront and the colour code is the normalized value of the instantaneous reciprocity gap at this position.

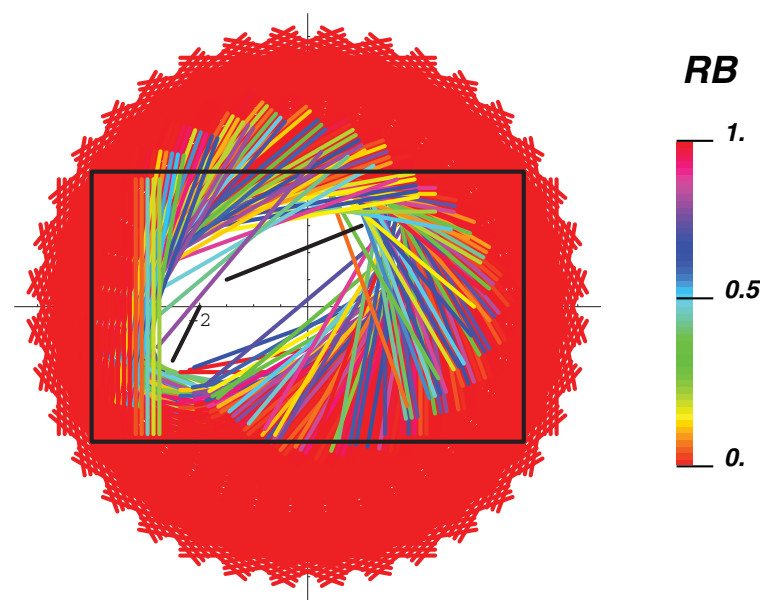

Figure 6. The 'convex hull' obtained for two cracks with the technique of the instantaneous reciprocity gap. Each line segment represents the position of the wavefront and the colour code is the normalized value of the instantaneous reciprocity gap at this position.

defines the region where the interaction between the adjoined wave and the displacement jump on the crack is negligible. As a consequence this domain defines together with the fronts of the adjoined wave at each time location, a sort of 'convex hull' of the crack. This representation is compared in figures 5 and 6 with the position of the real crack.

The small errors at the end of the crack have the length order of one finite element. This is due to the fact that the displacement jump is nonzero only at the first node after the crack tip. As such the virtual work measured by the reciprocity is nonzero only after a penetration of the adjoint wave of more than one element in the crack region.

One can remark that both techniques have given a very good match of the identified position and extension of the crack with the real crack. 


\section{Discussion of boundary conditions in earthquake problems}

The preceding permits us to identify in a simple way the displacement jump on an internal crack using the instantaneous reciprocity gap and a well-chosen adjoined wave. We shall analyse next the specific crack boundary conditions in the case of an earthquake in order to show that the same method can be applied in the identification of fault slips.

Let us first recall that the complete expression of the reciprocity gap obtained without using the information about a stress-free crack surface is the following:

$$
\mathcal{R B}=\int_{0}^{\infty} \int_{\Gamma_{C}} \llbracket u \rrbracket \cdot \sigma[\boldsymbol{w}] \cdot \boldsymbol{n}-\boldsymbol{w} \cdot \llbracket \sigma[u] \rrbracket \cdot n \mathrm{~d} s \mathrm{~d} t .
$$

In the case of an earthquake the boundary conditions on the crack corresponding to (5) have a slightly modified form. Let us consider an orthogonal system of coordinates on the fault $\Gamma_{C}$ given by the tangent and normal unit vectors $\left(\boldsymbol{t}_{1}, \boldsymbol{t}_{2}, \boldsymbol{n}\right)$. We shall consider the tangent and normal components of the displacement and traction vectors on the fault:

$$
\begin{aligned}
& \boldsymbol{u}=u_{1} \boldsymbol{t}_{1}+u_{2} \boldsymbol{t}_{2}+u_{n} \boldsymbol{n} \\
& \boldsymbol{\tau}=\boldsymbol{\sigma} \cdot \boldsymbol{n}=\tau_{1} \boldsymbol{t}_{1}+\tau_{2} \boldsymbol{t}_{2}+\tau_{n} \boldsymbol{n} .
\end{aligned}
$$

The crack $\Gamma_{C}$ represents in this case the geological fault and is prestressed before the earthquake, for $t<0$ :

$$
\begin{array}{ll}
\llbracket u_{1} t_{1}+u_{2} t_{2} \rrbracket=0 & \llbracket u_{n} \rrbracket=0 \\
\llbracket \tau_{1} t_{1}+\tau_{2} t_{2} \rrbracket \neq 0 & \llbracket \tau_{n} \rrbracket=0 .
\end{array}
$$

The earthquake actually releases the tangential shear stress in the slip of the fault, which implies for $t \geqslant 0$ under the hypothesis of a frictionless slip:

$$
\begin{array}{ll}
\llbracket u_{1} \boldsymbol{t}_{1}+u_{2} \boldsymbol{t}_{2} \rrbracket \neq 0 & \llbracket u_{n} \rrbracket=0 \\
\tau_{1}^{ \pm} \boldsymbol{t}_{1}+\tau_{2}^{ \pm} \boldsymbol{t}_{2}=0 & \llbracket \tau_{n} \rrbracket=0 .
\end{array}
$$

The following expression for the reciprocity gap reduces then to the following expression:

$$
\mathcal{R B}=\int_{0}^{\infty} \int_{\Gamma_{C}} \llbracket u_{1} \boldsymbol{t}_{1}+u_{2} \boldsymbol{t}_{2} \rrbracket \cdot\left(\tau_{1}[\boldsymbol{w}] \boldsymbol{t}_{1}+\tau_{2}[\boldsymbol{w}] \boldsymbol{t}_{2}\right) \mathrm{d} s \mathrm{~d} t
$$

and as such measures the mechanical work of the adjoint stresses on the tangential slip on the crack.

If the slip involves friction then there is a continuity of the tangential traction vector on the fault:

$$
\llbracket \tau_{1} t_{1}+\tau_{2} t_{2} \rrbracket=0
$$

and we obtain the same expression for the reciprocity gap $\mathcal{R B}$.

The specific boundary conditions in the case of fault slip in an earthquake reduce the expression of the reciprocity gap to the jump of the tangential displacement. Reviewing that the assumption of the preceding identification method still applies in this case. A further numerical study, taking into account no interpenetration and friction on the crack during the measurement simulations should validate the robustness of the identification method in this case. 


\section{Conclusion}

The previous results show that a simple direct interpretation of the instantaneous reciprocity gap leads to very precise identification of linear cracks in two-dimensional elastodynamic problems. The present technique can easily be applied to three or higher dimensional spaces. Moreover, it has also been shown that the method should also apply to problems with contact boundary conditions, such as those corresponding to earthquake problems. A complete analysis of this case is the subject of future work.

The numerical results are very promising and one can expect that other types of convex defects can also be identified using the same technique in the near future. As an illustration of this fact we presented in figure 6 the identification of two cracks in the same configuration as those discussed in this work.

\section{References}

[1] Andrieux S, Ben Abda A and Bui H D 1997 Sur l'identification de fissures planes via le concept d'écart à la réciprocité en élasticité $C$. R. Acad. Sci., Paris 324 1431-8

[2] Andrieux S, Ben Abda A and Bui H D 1999 Reciprocity principle and crack identification Inverse Problems 15 59-65

[3] Alves C J S and Ha-Duong T 1999 Inverse scattering for elastic plane waves Inverse Problems 15 91-7

[4] Ben Abda A and Bui H D 2003 Planar crack identification for the transient heat equation J. Inv. Ill-Posed Problems 11 27-31

[5] Bui H D, Constantinescu A and Maigre H 1999 Diffraction acoustique inverse de fissure plane: solution explicite pour un solide borné C. R. Acad. Sci., Paris 327 971-6

[6] Calderon A 1980 On an inverse boundary problem Seminar on Numerical Analysis and Application to Continuum Physics (Rio de Janeiro) pp 65-73

[7] Grasselli M and Yamamoto M 1998 Identifying a spatial body force in linear elastodynamics via traction measurements SIAM J. Control Optim. 36 1190-206 